\title{
$N$-boson spectrum from a discrete scale invariance
}

\author{
A. Kievsky, ${ }^{1}$ N. K. Timofeyuk, ${ }^{2}$ and M. Gattobigio ${ }^{3}$ \\ ${ }^{1}$ Istituto Nazionale di Fisica Nucleare, Largo Pontecorvo 3, 56100 Pisa, Italy \\ ${ }^{2}$ Department of Physics, University of Surrey, Guildford, Surrey GU2 7XH, United Kingdom \\ ${ }^{3}$ Université de Nice-Sophia Antipolis, Institut Non-Linéaire de Nice, CNRS, 1361 route des Lucioles, O6560 Valbonne, France
}

(Received 9 May 2014; published 4 September 2014)

\begin{abstract}
We present an analysis of the $N$-boson spectrum computed using a soft two-body potential, the strength of which has been varied in order to cover an extended range of positive and negative values of the two-body scattering length $a$ close to the unitary limit. The spectrum shows a tree structure of two states, one shallow and one deep, attached to the ground state of the system with one less particle. It is governed by a unique universal function $\Delta(\xi)$, already known in the case of three bosons. In the three-particle system the angle $\xi$, determined by the ratio of the two- and three-body binding energies $E_{3} / E_{2}=\tan ^{2} \xi$, characterizes the discrete scale invariance of the system. Extending the definition of the angle to the $N$-body system as $E_{N} / E_{2}=\tan ^{2} \xi$, we study the $N$-boson spectrum in terms of this variable. The analysis of the results, obtained for up to $N=16$ bosons, allows us to extract a general formula for the energy levels of the system close to the unitary limit. Interestingly, a linear dependence of the universal function as a function of $N$ is observed at fixed values of $a$. We show that the finite-range nature of the calculations results in the range corrections that generate a shift of the linear relation between the scattering length $a$ and a particular form of the universal function. We also comment on the limits of applicability of the universal relations.
\end{abstract}

DOI: 10.1103/PhysRevA.90.032504

PACS number(s): 31.15.xj, 67.85.-d, 03.75.-b, 21.45.-v

\section{INTRODUCTION}

Physical systems present universal behavior when specific details of the interaction between their constituents are suppressed in favor of a few control parameters that determine the dynamics. Well-known examples of this kind are critical phenomena in which the systems, which are very different at the microscopic level, show a set of equal critical exponents. Around the critical point the dynamics is governed by longrange correlations and not by the details of the interaction between the constituents. When a universal class is identified all systems belonging to this class can be described equally well by a model in which this particular phenomenon is implemented. An example is the use of the Ising model to study phase transitions.

Here we analyze a particular universal behavior of fewboson systems having a large two-body scattering length. In two-body systems, the universality is governed by one parameter, the scattering length $a$. When $a$ is large and positive the two-body system has a shallow bound state with an energy of $E_{D} \approx \hbar^{2} / m a^{2}$ (shallow dimer) and, in addition, all the low-energy observables are governed by $a$. The system has a continuous scale invariance that strongly constrain the form of the observables. This symmetry is broken in the $s$-wave threebody sector. However, the three-body system still has a residual symmetry, the discrete scale invariance (DSI), meaning that the physics is invariant under the rescaling $r \rightarrow \Lambda^{n} r$, where the constant $\Lambda$ is usually written as $\Lambda=e^{\pi / s_{0}}$, with $s_{0} \approx 1.00624$ being a universal number that characterizes a system of three identical bosons (for a recent review see Ref. [1]).

As has been shown by Efimov [2,3], in the limit of large scattering length $a \rightarrow \infty$ (unitary limit), the threeboson spectrum consists of an infinite number of states that accumulate to zero with the ratio between the energies of two consecutive states being $E_{3}^{n+1} / E_{3}^{n}=e^{-2 \pi / s_{0}}$. This is known as the Efimov effect and its characteristics have been the subject of intense investigation both experimentally [4-7] and theoretically [8-12]. In recent years the study of the Efimov effect has been extended to what is now called Efimov physics and refers to the physics of shallow states. In these states the particles stay mostly far apart from each other, with the consequence that the dynamics is largely insensitive to the details of the interaction.

In the case of bound states, the three-boson spectrum in the limit of a zero-range interaction in the two-body subsystem (zero-range limit or scaling limit) can be expressed in the parametric form

$$
\begin{gathered}
E_{3}^{n} /\left(\hbar^{2} / m a^{2}\right)=\tan ^{2} \xi, \\
\kappa_{*} a=e^{\left(n-n^{*}\right) \pi / s_{0}} \frac{e^{-\Delta(\xi) / 2 s_{0}}}{\cos \xi},
\end{gathered}
$$

where $\kappa_{*}$ is the wave number corresponding to the energy of the $n^{*}$ level at the unitary limit and is called the three-body parameter. The function $\Delta(\xi)$ is a universal function and its parametrization in the range $[-\pi,-\pi / 4]$ is given in Ref. [1]. Equation (1b) explicitly manifests DSI: The ratio $E_{3}^{n+1} / E_{3}^{n}$ remains constant at each value of the angle $\xi$ and is equal to $e^{-2 \pi / s_{0}} \approx 1 / 515.03$. Another characteristic of the above equation is that the three-boson spectrum is controlled by the two-body scattering length $a$ and it is completely determined by the knowledge of the three-body parameter $\kappa_{*}$, which appears as a scale parameter. Many examples of how Eqs. (1) are used can be found in the literature (see, for example, Refs. [1,13]).

In the four-body case, it has been shown that two levels $E_{4}^{n, 0}$ and $E_{4}^{n, 1}$ appear attached to each $E_{3}^{n}$ level [9-12,14,15]. Moreover, at the unitary limit the ratios $E_{4}^{n, 0} / E_{3}^{n} \approx 4.611$ and $E_{4}^{n, 1} / E_{3}^{n} \approx 1.002$ are universal and their numerical values have been estimated using different approaches. In Ref. [16] it was shown that universal ratios also exist at the universal limit in systems with $N \leqslant 13$ bosons and these ratios were estimated by solving the corresponding Schrödinger equation with finite-range two-body potentials. 
In Ref. [17] the spectra and universality of small helium clusters up to $N=6$ were studied in the $(1 / a, \kappa)$ plane, where $\kappa=\operatorname{sgn}(E)\left[|E| /\left(\hbar^{2} / m\right)\right]^{1 / 2}$ and $E$ is the energy of a level. The two lowest levels with the energies $E_{N}^{0,0}$ and $E_{N}^{0,1}$, attached to the ground state of the $N-1$ system, with energy $E_{N-1}^{0,0}$, were observed in the region of $a$ studied. Furthermore, in Refs. [16,17] the values of $a$ at which the $N$-body cluster disappears into the $N$-body continuum were estimated.

The examples of universal ratios (the correlations between observables) discussed above can be complemented by the Phillips and the Tjon lines [18,19] studied in nuclear physics to analyze the correlations between the triton binding energy and the doublet $n$ - $d$ scattering length and between the triton and $\alpha$ particle binding energies, respectively. These correlations indicate a limitation in the number of scales that governs the dynamics of the system [12].

In the present work we address the constraints imposed by the DSI in the spectrum of the $N$-boson system with a general number of bosons $N$. In addition, we will answer the following questions. (i) Is the tree structure of two states $E_{N}^{0,0}$ and $E_{N}^{0,1}$ attached to the $E_{N-1}^{0,0}$ state and observed up to $N=6$ valid for general values of $N$ ? (ii) Are there any general relations for the ratios $E_{N}^{n, 0} / E_{N-1}^{n, 0}$ and $E_{N}^{n, 1} / E_{N-1}^{n, 0}$ ? We answer these question by analyzing the spectra of $N \leqslant 16$ particles obtained from solution of the many-body Schrödinger equation with a soft finite-range two-body force.

The paper is organized as follows. The working equations determining the spectrum of the $N$-boson system in the zerorange limit are given in Sec. II, while in Sec. III we introduce their finite-range version. The analysis of the numerical results is given in Sec. IV and the DSI for $N$ bosons is analyzed in Sec. V. In Sec. VI the equations proposed in the preceding sections are used to analyze selected experimental as well as theoretical results from the literature. In Sec. VII a summary is given.

\section{THE $N$-BOSON SYSTEM IN THE ZERO-RANGE LIMIT}

Our aim is to discuss an extension of Eqs. (1), which describes the energy spectrum of the three-boson system close to the unitary limit in the zero-range limit, to $N>3$. This extension is based on the detailed analysis of a four-boson system with a large two-body scattering length, reported first in Ref. [9] and then in Refs. [10,11], and on an extended analysis of DSI in Ref. [20]. It was found in Ref. [9] that the four-body system has two bound states, one of which is deeply bound and another that is shallow very close to the threshold of disintegration into one boson and a trimer. Using a DSI argument, it has been conjectured that the two-level structure is tied to each three-body state. However, only the lowest two states, attached to the trimer bound state $E_{3}^{0}$, are true bound states; the other ones appear as resonances since they are above the trimer-particle threshold. The study of this particular tree structure was analyzed in Ref. [10], in which the notation $E_{4}^{n, m}$ was proposed to identify the energy of each four-body level. In this notation, $n$ indicates a three-body level and $m=0$ identifies the deep state while $m=1$ labels the shallow state. The fact that this structure of levels results from a DSI can be seen from the universal character of the ratio
$E_{4}^{n, m} / E_{3}^{n}$. A study of this ratio at the unitary limit was done in Refs. [10,11] with the conclusion that $E_{4}^{n, 0} / E_{3}^{0} \approx 4.611$ and $E_{4}^{n, 1} / E_{3}^{0} \approx 1.002$. A more extended analysis of the DSI can be done studying these ratios along the $(1 / a, \kappa)$ plane at fixed values of the angle $\xi$ (see Ref. [20]). Moreover, a recent work [21] has shown that the universal function $\Delta(\xi)$, which governs the three-boson dynamics, is also responsible for the $N$-boson dynamics. All these findings suggest the following extension of Eqs. (1) to $N \geqslant 4$ :

$$
\begin{gathered}
E_{N}^{n, m} /\left(\hbar^{2} / m a^{2}\right)=\tan ^{2} \xi, \\
\kappa_{N}^{m} a=e^{\left(n-n^{*}\right) \pi / s_{0}} \frac{e^{-\Delta(\xi) / 2 s_{0}}}{\cos \xi}, x
\end{gathered}
$$

with $E_{N}^{n, m}$ the deep $(m=0)$ or shallow $(m=1) N$-body state attached to the $n$th Efimov trimer.

Equations (2) have the remarkable property that the $N$-boson spectrum is controlled by the two-body scattering length, by the universal function $\Delta(\xi)$, and that it is completely determined by the knowledge of $\kappa_{N}^{m}$. The $\kappa_{N}^{m}$ are not true independent parameters; they are fixed by the three-body scale $\kappa_{*}$. For instance, in Ref. [21] it was shown that

$$
\kappa_{N}^{0} / \kappa_{*}=1+1.147(N-3) .
$$

This result was obtained by observing that $\kappa_{N}^{m}$ is a linear function of $N$ and using the universal ratio given in Ref. [11]. In the following we analyze the spectrum of $N$-boson systems up to $N=16$ in order to extend this relation to $m=1$ and, from a more general perspective, to verify the validity of Eqs. (2).

\section{FINITE-RANGE CORRECTIONS}

The spectrum of the three-boson system, obtained by solving the Schrödinger equation with soft two-body potentials close to the unitary limit, was analyzed in Refs. [22,23]. It was shown that Eqs. (1) have to be modified in order to take into account the finite-range character of those calculations. Based on the analysis of the energies of the three-body system obtained numerically, the following modified equations have been deduced:

$$
\begin{aligned}
E_{3}^{n} / E_{2} & =\tan ^{2} \xi \\
\kappa_{3}^{n} a_{B}+\Gamma_{3}^{n} & =\frac{e^{-\Delta(\xi) / 2 s_{0}}}{\cos \xi} .
\end{aligned}
$$

Despite some similarities, there are several important differences from the zero-range theory of Eqs. (1).

(i) The parameters $\kappa_{3}^{n}$ carry explicitly the index $n$ labeling the different tree branches since the ratio $\kappa_{3}^{n} / \kappa_{3}^{n+1}$ for two successive branches is in general slightly different from the scaling factor $e^{\pi / s_{0}}$. In fact, these ratios include finite-range corrections and their specific values can be extracted from the numerical solutions. The correspondence between Eqs. (1) and (4) is made by identifying the scale parameter $\kappa_{*}$ with one of the parameters $\kappa_{3}^{n}$. For example, in Ref. [22] the threehelium-atom case was studied as a reference system and $\kappa_{*}$ was identified with $\kappa_{3}^{1}$, the branch corresponding to the first excited state. 
(ii) The quantity $\hbar^{2} / m a^{2}$ is replaced by $E_{2}=\hbar^{2} / m a_{B}^{2}$, which is the dimer binding energy in the case of positive scattering length $a$ or, for negative values of $a$, the two-body virtual-state energy. Also in Eq. (4b) $a$ is replaced by the scattering length $a_{B}$ corresponding to finite-range two-body potential. The replacement of $a$ by $a_{B}$ introduces some range corrections as the value of $a$ moves away from the unitary limit. It should be noted that the relation $E_{2}=\hbar^{2} / m a^{2}$ is exact in the case of zero-range two-body interactions.

(iii) The main modification in Eqs. (4) is the introduction of the shift parameter $\Gamma_{3}^{n}$. The origin of the shift was discussed in Ref. [21], where it was shown that it essentially appears from the first-order expansion of the scaling-violating momentum $\Lambda_{0}$, in terms of powers of $r_{0} / a$, where $r_{0}$ is the interaction range. The parameter $\Lambda_{0}$ fixes the value of the logarithmic derivative of the wave function close to the origin and encodes the short-range physics [1]. In the zero-range model one can identify $\Lambda_{0}$ with the three-body parameter $\kappa_{*}$ leading directly to Eqs. (1). However, for finite-range potentials the $\Lambda_{0}=\kappa_{*}$ relation does not hold anymore and we propose the finite-range correction

$$
\Lambda_{0}=\kappa_{*}\left(1+\mathcal{A} \frac{r_{0}}{a}+\cdots\right),
$$

leading directly to Eqs. (4), with $\Gamma_{3}^{0}=\mathcal{A} \kappa_{3}^{0} r_{0}$ (here we assume $\kappa_{*}=\kappa_{3}^{0}$ ). This can be proved by making use of Eqs. (187) and (193) from Ref. [1].

As a function of the finite-range-corrected scattering length $a_{B}$, Eq. (4b) is a two-parameter equation: the scale parameter $\kappa_{3}^{n}$ and the finite-range parameter $\Gamma_{3}^{n}$. As in the study of critical phenomena, $\kappa_{3}^{n}$ can be interpreted as a material-dependent parameter and $\Gamma_{3}^{n}$ as the analog of the finite-scale correction (here finite-range correction). Their introduction allows the collapse of observables onto a single universal curve [24]. In fact, in Ref. [21] it was shown that plotting $E_{3}^{n} / E_{2}$ in terms of $1 /\left(\kappa_{3}^{n} a_{B}+\Gamma_{3}^{n}\right)$ makes the calculated points collapse onto a universal curve.

The extension of Eqs. (4) to general $N$ has been proposed in Ref. [21] where the spectrum of the $N$-boson system, obtained by solving the Schrödinger equation with soft potentials close to the unitary limit, was analyzed up to $N=6$. The results of that work show that Eqs. (2) have to be modified in order to take into account the finite-range character of those calculations. The general form is suggested,

$$
\begin{aligned}
E_{N}^{n, m} / E_{2} & =\tan ^{2} \xi, \\
\kappa_{N}^{n, m} a_{B}+\Gamma_{N}^{n, m} & =\frac{e^{-\Delta(\xi) / 2 s_{0}}}{\cos \xi},
\end{aligned}
$$

and applied to the lowest tree of the two-level structure, which corresponds to $n=0$. The index $n$ identifies the $N=3$ branch and the index $m$ takes the values $m=0,1$. The parameters $\kappa_{N}^{n, m}$ are the energy wave numbers at the unitary limit $\left(\kappa_{N}^{n, m}\right)^{2} \hbar^{2} / m=E_{N}^{n, m}$ and explicitly show the index $n$ labeling the different $N=3$ branches due to the finite-range corrections in $\kappa_{3}^{n}$.

It should be stressed that, for $N>3$, the $n=0$ case is of particular interest since it describes bound states. In the next section we analyze the $n=0$ branch, extracting the $\kappa_{N}^{0, m}$ values from the numerical solutions and showing that they have a linear dependence on $N$ with a slope slightly different from the one suggested by the zero-range theory. We show that, as $a$ moves from the unitary limit toward lower positive values, the validity of Eqs. (6) could be limited. The system becomes more compact, losing its universal character. In the case of $n>0$ the validity of Eqs. (6) is limited by the appearance of different thresholds as the positive values of $a$ decrease. For example, in Ref. [25] it was shown that, in the $N=4$ case, a shallow tetramer decays at the atom-trimer threshold, becoming an inelastic virtual state. In the present work we limit the discussion of Eqs. (6), in the case of $N>3$, to the case of bound states (the $n=0$ branch).

\section{ANALYSIS OF $N$-BODY SOLUTIONS}

To study the validity of Eqs. (2) and (6) we follow Ref. [17] and describe the $N$-boson system using a two-body Gaussian (TBG) potential

$$
V(r)=V_{0} e^{-r^{2} / r_{0}^{2}} .
$$

We solve the $N$-body Schrödinger equation with mass parameter $\hbar^{2} / m=43.281307\left(a_{0}\right)^{2} \mathrm{~K}$. Using $r_{0}=10 a_{0}$ and $V_{0}=$ $-1.2343566 \mathrm{~K}$, the model reproduces the binding energy and the scattering length of two helium atoms described by a widely used He-He interaction, the LM2M2 potential [26], which has a van der Waals length $\ell=10.2 a_{0}$.

To solve the Schrödinger equation for $N$ bosons we use the hyperspherical harmonic method in the version proposed in Ref. [27]. This method reproduces the values given in Ref. [28] up to $N=6$ and here we extend the calculations up to $N=16$. Increasing the grand angular quantum number $G$, we obtain converged results for the ground state and first excited state of the $N$-boson systems. As discussed in Refs. [27,29], convergence of the ground-state energy is obtained with relatively low values of $G$; with values of $G \leqslant 12$ an accuracy greater than $1 \%$ is obtained. In the case of the first excited state a similar accuracy would need a much higher value of $G$, making the computation of this state very difficult. In the present work we use the results with $G \leqslant 12$ to extrapolate the first excited energy with an accuracy of a few percent.

Varying the strength $V_{0}$ of the TBG potential (7) we explore the $\left(a^{-1}, \kappa\right)$ plane. For each value of the potential strength $V_{0}$ we determine $E_{2}$ and $a$ and then compute the energies of the ground and first excited states of the $N$-body systems. For $N=3$ we compute $E_{3}^{0}$ and $E_{3}^{1}$, the values that define the first two energy branches with $n=0$ and 1 . For $N>3$ we compute the two-level structure of the $n=0$ branch. We denote these states by $E_{N}^{0}$ and $E_{N}^{1}$, omitting the index $n$ from now on, and we analyze the two-level spectrum up to $N=16$.

Using Eqs. (4a) and (6a), we determine the value of the angle $\xi$ from which we can compute the universal function $y(\xi)$,

$$
y(\xi)=\frac{e^{-\Delta(\xi) / 2 s_{0}}}{\cos \xi},
$$

appearing on the right-hand side of Eqs. (4b) and (6b). The function $y(\xi)$ has a linear dependence on $a_{B}$ for each value of $N$ and $m$. In fact, Eq. (6b) can be rewritten as

$$
y=\kappa_{N}^{m} a_{B}+\Gamma_{N}^{m} .
$$




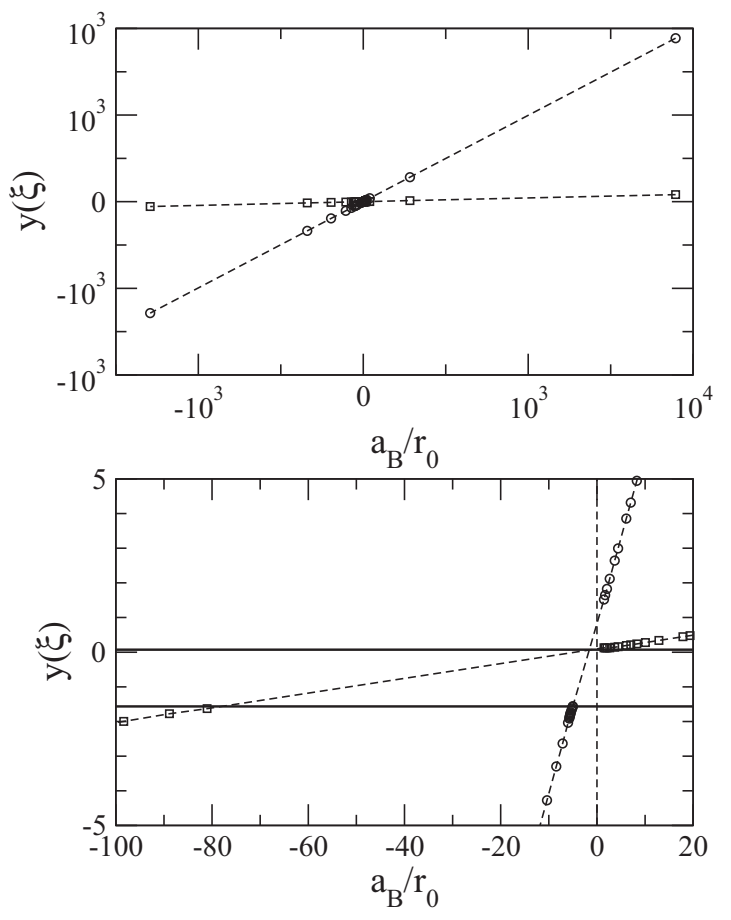

FIG. 1. Universal function $y(\xi)$ as a function of $a_{B}$ in units of $r_{0}$. Numerical results are given by circles $(n=0)$ and squares $(n=1)$ with the dashed lines representing the best linear fits to the results (upper panel). The bottom panel shows a zoom close to the thresholds $y(-\pi)=-1.56$ and $y(-\pi / 4)=0.071$ (given by the thick horizontal lines).

In the case of a zero-range interaction $a_{B}=a$ and $\Gamma_{N}^{m}=0$ and the linear relation results in $y=\kappa_{N}^{m} a$, representing a straight line passing through the origin with the slope determined by the value of $\kappa_{N}^{m}$. In the case of a finite-range interaction the linear dependence between $y$ and $a_{B}$ remains, but the straight line does not go through the origin.

To make a connection with previous analysis, we first present our results for the $N=3$ case. Their plot in the $\left(a^{-1}, \kappa\right)$ plane have been reported many times in the literature. Here we prefer to use the $\left(a_{B}, y\right)$ plane, stressing the linear relation between $a_{B}$ and $y$ that follows from Eq. (9). The results are shown in Fig. 1 as circles (the $n=0$ branch) and squares (the $n=1$ branch). The dashed lines represent the best linear fit to the results, which can be parametrized with $\kappa_{3}^{0}=0.0488 a_{0}{ }^{-1}$, $\Gamma_{3}^{0}=0.869$ and $\kappa_{3}^{1}=0.00212 a_{0}{ }^{-1}, \Gamma_{3}^{1}=0.0840$. The fit has $\chi^{2} \approx 0.1$, proving that the behavior of the numerical results is in very good agreement with a linear dependence. Moreover, at the unitary limit the extracted values for $\kappa_{3}^{n}$ coincide up to four figures (or better) to the calculated values. The DSI predicts in the zero-range limit that $\kappa_{3}^{0} / \kappa_{3}^{1}=e^{\pi / s_{0}} \approx 22.7$, while from our results we obtain a slightly different value, $\kappa_{3}^{0} / \kappa_{3}^{1} \approx 23.0$. This change is due to the finite-range character of the two-body interaction.

The top panel of Fig. 1 shows all calculated $y(\xi)$ values plotted within a very extended range of $a_{B}$. The bottom panel displays a zoom around the thresholds $y(-\pi) \approx-1.56$, at which the trimer levels disappear into the three-body continuum (for a more precise value see Ref. [30]), and $y(-\pi / 4) \approx 0.071$, at which the trimer disappears on the particle-dimer continuum (the thresholds are shown by thick horizontal lines). Close to the threshold at -1.56 we observe a strong linear trend in the $y(\xi)$ behavior as a function of $a_{B}$. This allows us to extract with great confidence the (negative) values of the scattering length $a_{3}^{0,-}$ and $a_{3}^{1,-}$ at which the trimer ground state $E_{3}^{0}$ and first excited state $E_{3}^{1}$ disappear into the three-atom continuum. Using the values of $\kappa_{3}^{n}$ and $\Gamma_{3}^{n}$ from the linear fit and transforming $a_{B}$ to $a$, we obtain $a_{3}^{0,-} \approx-44 a_{0}$ a.u. and $a_{3}^{1,-} \approx-745 a_{0}$, which are in close agreement with the estimates given in Ref. [17].

The threshold at 0.071 indicates the point at which the bound state disappears in the atom-dimer continuum. We cannot reach it by lowering the scattering length because as $a \rightarrow r_{0}$ the three-body states become more bound, leaving the Efimov window. For the case of the excited state this is less obvious, but in the proximity to the atom-dimer threshold our results lie on a line parallel to (and slightly above) the $y(-\pi / 4)$ line without crossing it. This has been clearly seen also in Ref. [17], where the first excited state for three helium atoms has been shown not to cross the atom-dimer threshold but move almost parallel to it from below (see also Ref. [31]).

The analysis of the Efimov trimers in the $\left(a_{B}, y\right)$ plane has shown that they can be described by straight lines defined by the parameters $\kappa_{3}^{n}, \Gamma_{3}^{n}$, and $a_{3}^{n,-}$. Next we study the $N \geqslant 4$ results for $y(\xi)$ in the same $\left(a_{B}, y\right)$ plane. These results are presented by circles in Fig. 2 , which, as in the $N=3$ case, can be fitted by straight lines shown as solid lines in this figure. Figures 2(a) and 2(b) show the results for the ground state $(m=0)$ and the first excited state $(m=1)$, respectively. Figures 2(c) and 2(d) zoom the area around the threshold $y(-\pi)=-1.56$ (shown as a thick line). In the case of $N=4,5,6$, very detailed calculations have been done close to this threshold.

A remarkable characteristic of Fig. 2 is that the straight lines cross each other almost in one single point, the position of which is slightly different for the ground and excited states. To study further this fact we extract the values of $\kappa_{N}^{m}$ from the slopes of the straight lines. They coincide up to four significant figures with the values calculated at the unitary limit given by $V_{0} \approx-1.162 \mathrm{~K}$. The results are given in Fig. 3, where we show the values of $\kappa_{N}^{0}$ (circles) and $\kappa_{N}^{1}$ (squares) as a function of $N$ [Fig. 3(a)] and the values of $\Gamma_{N}^{0}$ (circles) and $\Gamma_{N}^{1}$ (squares) [Fig. 3(b)]. The $\kappa_{N}^{0}$ and $\kappa_{N}^{1}$ form two almost parallel lines, whereas $\Gamma_{N}^{0}$ and $\Gamma_{N}^{1}$ collapse in one line as $N$ increases. However, both $m=0$ and 1 explicitly show a linear dependence on $N$, which is illustrated in Fig. 4, where the best linear fits to the data are shown by the solid lines and the circles (squares) corresponds to $m=0(m=1)$. Using this linear relation, we extract the coordinates of the point $\left(a_{B}^{m}, \Gamma_{m}\right)$ at which the straight lines corresponding to the ground state $m=0$ and excited state $m=1$ cross each other. Defining $\Gamma_{N}^{m}=\Gamma_{m}-\kappa_{N}^{m} a_{B}^{m}$, we obtain the relation between $\kappa_{N}^{m}$ and $y$ :

$$
\kappa_{N}^{m}\left(a_{B}-a_{B}^{m}\right)+\Gamma_{m}=y(\xi) .
$$

From the analysis of Fig. 4 we get $a_{B}^{0}=7.077 a_{0}$ and $\Gamma_{0}=0.768$ for the ground states and $a_{B}^{1}=7.304 a_{0}$ and $\Gamma_{1}=$ 0.887 for the excited states. A consequence of the different locations of these two points is that the line corresponding to the shallow state $E_{N}^{1}$ can cross the line of the ground 

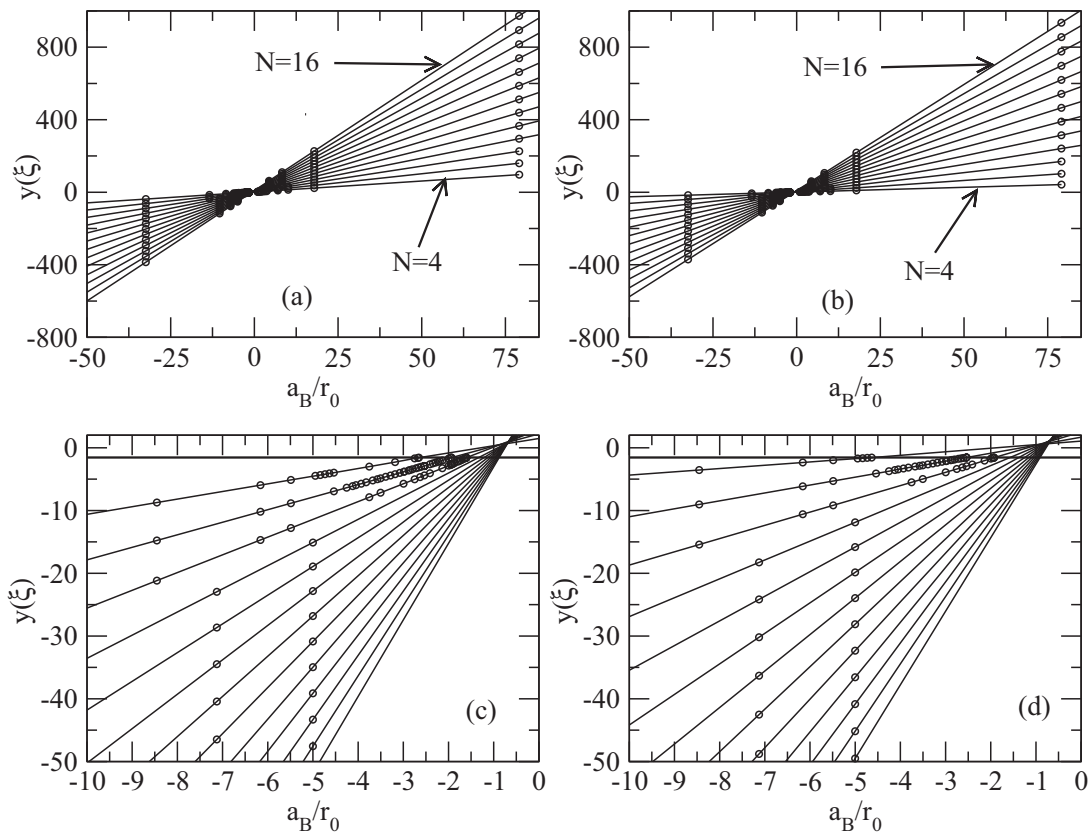

FIG. 2. Universal function $y(\xi)$ as a function of $a_{B}$, in units of $r_{0}$ for $N=4-16$ for (a) ground-state energies and (b) excited-state energies. A zoom of the plots close to the -1.56 threshold is given for (c) $n=0$ and (d) $n=1$. state $E_{N-1}^{0}$, resulting in an unbound excited state. This is shown in Fig. 5, where one can see that starting with $N=8$ the $E_{N}^{1}$ excited state is no longer bound at the $y(-\pi)$ threshold.

Equation (10) can be further simplified by taking into account the linear relation between $\kappa_{N}^{m}$ and $N$. In fact, as has been inferred in Ref. [21] and from the analysis of Fig. 3, the relation can take the form

$$
\kappa_{N}^{m}=\kappa_{4}^{m}+(N-4)\left(\kappa_{5}^{m}-\kappa_{4}^{m}\right),
$$

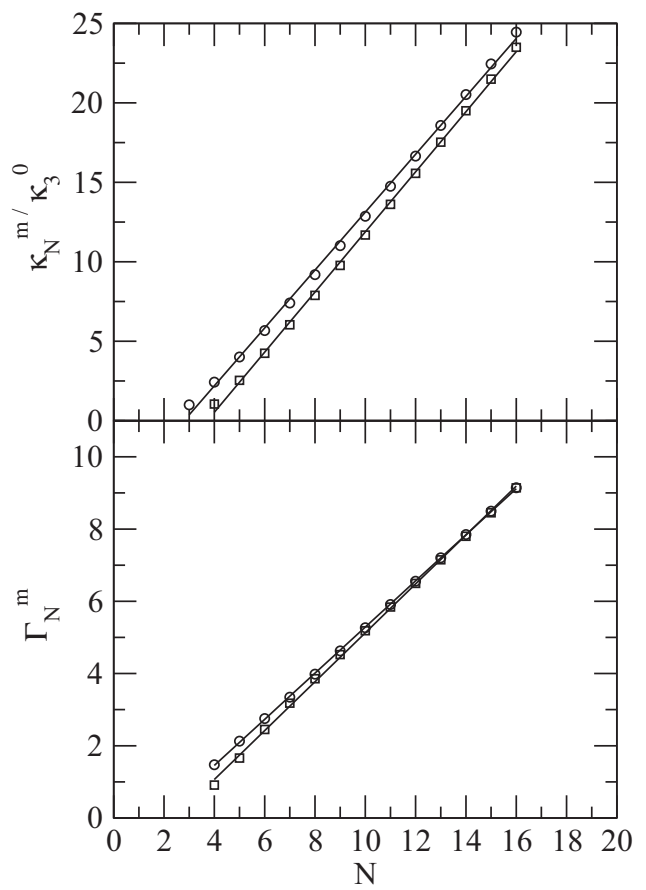

FIG. 3. Ratio $\kappa_{N}^{m} / \kappa_{3}^{0}$ (top) and shift $\Gamma_{N}^{m}$ (bottom) as a function of $N$. The circles correspond to $m=0$ and the squares to $m=1$. The solid lines are the best linear fits. where we have used two points, corresponding to $N=4$ and 5 , to construct the line. However, other choices to describe the straight line produce similar results. With the above relation, a global description of the $m$ th level of the $N$-boson spectrum can be achieved with just four parameters. It should be stressed that the present results have been obtained using short-range two-body forces (or eventually three-body forces, as in Ref. [28]). The existence of high-order forces could modify the linear relation given in Eq. (11).

The main result of this analysis is the following. In order to describe the $m$ th energy level of the $N$-boson system, using short-range interactions, two parameters are needed, $\kappa_{N}^{m}$ and $\Gamma_{N}^{m}$. This description is particularly accurate near the unitary limit. It deteriorates at positive values of $a$ as $a \rightarrow \ell$, where the strength of the potential increases, making the $N$-body ground states deeper. In addition, as $N$ increases the finite character of the interaction allows new excited states to appear and the tree structure is lost. It was shown in Ref. [32] that, starting with $N \geqslant 12$, a second excited state appears at the

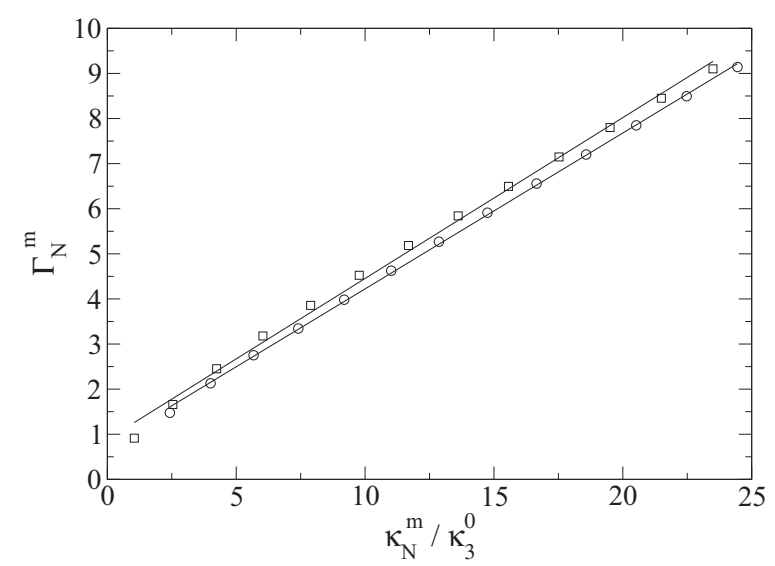

FIG. 4. Shift $\Gamma_{N}^{m}$ as a function of the ratio $\kappa_{N}^{m} / \kappa_{3}^{0}$ for $m=0$ (circles) and $m=1$ (squares). 


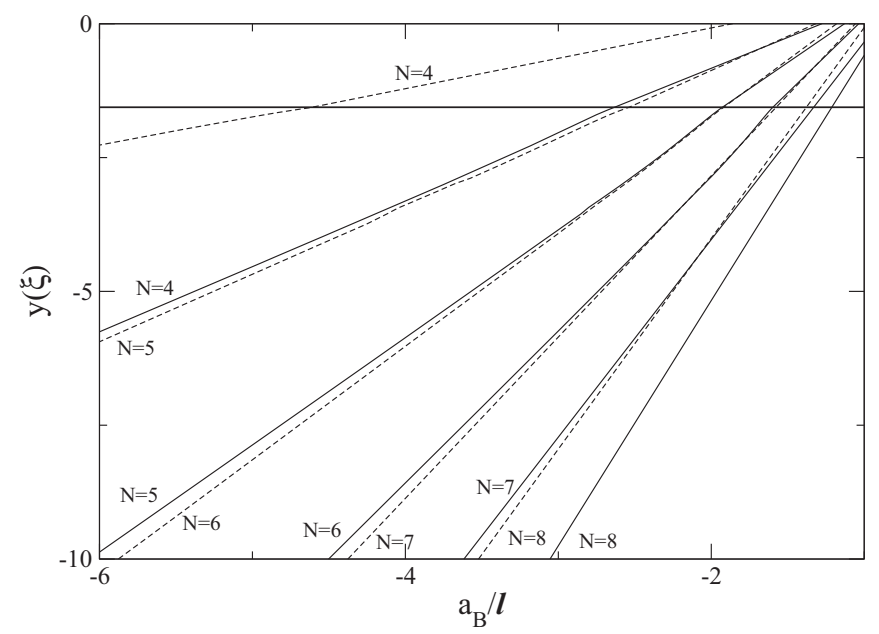

FIG. 5. Universal function $y(\xi)$ as a function of $a_{B}$, in units of $\ell$ for the ground state (solid lines) and excited state (dashed lines) close to the $y(-\pi)=-1.56$ threshold, for $N=4-8$. Starting with $N=8$, the excited state becomes unbound at this threshold as it crosses the ground-state line before arriving at the threshold.

unitary limit. However, the two-parameter description remains acceptable (of the order of a few percent or better) as the system approaches the $N$-body continuum (negative $a$ values), which corresponds to the best realization of shallow states, well described by the present formalism. Approaching the threshold on the $-\pi$ axis, all the excited states disappear for $N>7$, as has been discussed before. It should be noted that in the case of a zero-range interaction there is only one parameter $\kappa_{N}^{m}$ because $\Gamma_{N}^{m}=0$ and, as the crossing point is the origin for the two $m$ levels, the tree structure should remain valid with increasing $N$.

In the case of finite-range interactions, using the relation given by Eq. (11), a global fit of the $m$ th level is possible for general values of $N$ with only four parameters: two $\kappa_{N}^{m}$ values and the coordinates of the crossing point. However, this description is not as precise as the previous one and could introduce some errors. In the case of a zero-range interaction a global fit is possible with two parameters (two values of $\kappa_{N}^{m}$ ) and in this case the description should be exact. This is further analyzed in the next section.

\section{THE DSI AS A FUNCTION OF $N$}

The DSI in the $N=3$ system can be seen from the constant values of the ratio $E_{3}^{n} / E_{3}^{n^{\prime}}$ between two different branches at fixed values of the angle $\xi$. This property, in the zero-range theory, is encoded in Eqs. (1), from which it is easy to see that, when $\xi$ is constant, then $E_{3}^{n} / E_{3}^{n^{\prime}}=e^{2\left(n^{\prime}-n\right) \pi / s_{0}}$. Particular cases are the unitary limit corresponding to $\xi=-\pi / 2$ and the threshold at which the cluster disappear in the three-atom continuum corresponding to $\xi=-\pi$. However, that property holds in the range $-\pi \leqslant \xi \leqslant-\pi / 4$.

The extension of the zero-range theory to general $N$ is given in Eqs. (2). These equations explicitly state the following property for the ratio between two different branches with general values of $m$ at fixed values of $\xi$ :

$$
\frac{E_{N}^{n, m}}{E_{N^{\prime}}^{n^{\prime}, m^{\prime}}}=\left(\frac{\kappa_{N}^{m}}{\kappa_{N^{\prime}}^{m^{\prime}}}\right)^{2} e^{2\left(n-n^{\prime}\right) \pi / s_{0}} .
$$

Unlike in the $N=3$ case, no analytical expression for $\kappa_{N}^{m} / \kappa_{N^{\prime}}^{m^{\prime}}$ exists. It can only be determined from numerical analysis such as the one carried out in Ref. [11] for $E_{4}^{0} / E_{3}^{0}$. However, we can show that $\kappa_{N}^{0} / \kappa_{3}^{0}$ depends linearly on $\kappa_{4}^{0} / \kappa_{3}^{0}$ by using Eq. (11) twice, for $N=3$ and 4 . This results in the formula (see also Ref. [21])

$$
\frac{\kappa_{N}^{0}}{\kappa_{3}^{0}}=1+(N-3)\left(\frac{\kappa_{4}^{0}}{\kappa_{3}^{0}}-1\right),
$$

which in the zero-range limit reduces to $\kappa_{N}^{0} / \kappa_{3}^{0}=1+$ $1.147(N-3)$ when $\kappa_{4}^{0} / \kappa_{3}^{0}=2.147$ from Ref. [11] is used. Our numerical results using the TBG potential give $\kappa_{4}^{0} / \kappa_{3}^{0}=2.42$, showing some range corrections but not far from the zero-range limit. The square of Eq. (13) gives a quadratic dependence on $N$ of the $N$-boson ground-state energy $E_{N}^{0}$ expressed in terms of the three-boson ground-state energy. A quadratic relation in terms of $N$ has been also obtained in Ref. [33].

Our results can be used to study different ratios that might display universal character. In Fig. 6 we show the ratios between ground states $\kappa_{N+1}^{0} / \kappa_{N}^{0}$ (circles), between the shallow state of the $N+1$ system and the ground state of the $N$-body system $\kappa_{N+1}^{1} / \kappa_{N}^{0}$ (triangles), and the ratio between the shallow states $\kappa_{N+1}^{1} / \kappa_{N}^{1}$ (squares). At large $N$ the ratio $\kappa_{N+1}^{n} / \kappa_{N}^{n}$ tends to one, suggesting that

$$
\kappa_{N+1}^{0} / \kappa_{N}^{0} \approx \kappa_{N+2}^{1} / \kappa_{N+1}^{1} .
$$

This relation is a consequence of the almost constant behavior of the ratio $\kappa_{N+1}^{1} / \kappa_{N}^{0}$ (see Fig. 6) that allows Eq. (13) to be extended to shallow excited states of the tree structure for $N \geqslant 4$,

$$
\frac{\kappa_{N}^{1}}{\kappa_{4}^{1}}=1+(N-4)\left(\frac{\kappa_{5}^{1}}{\kappa_{4}^{1}}-1\right),
$$

which in the zero-range theory reduces to $\kappa_{N}^{1} / \kappa_{4}^{1}=1+$ $1.147(N-4)$. The third general ratio $\kappa_{N+1}^{1} / \kappa_{N}^{0}=\kappa_{4}^{1} / \kappa_{3}^{0}$ can

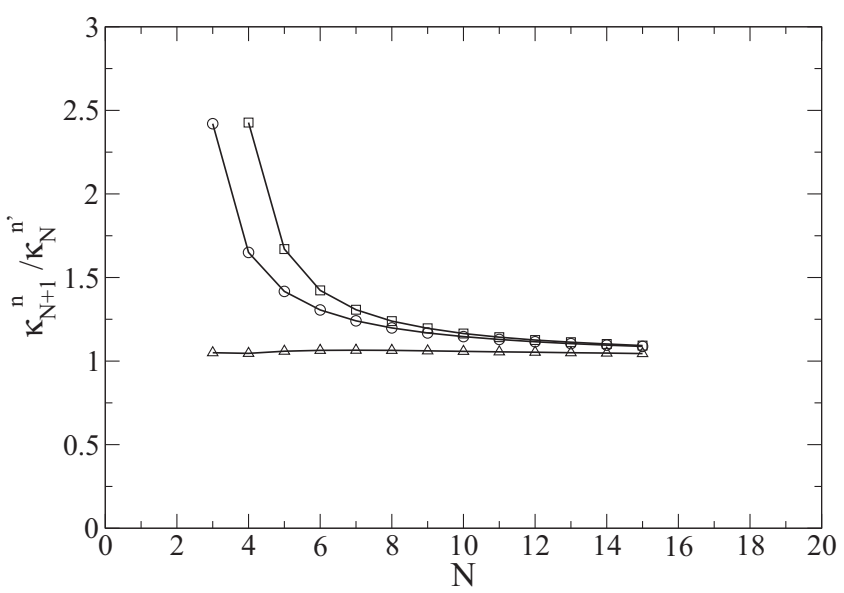

FIG. 6. Ratios $\kappa_{N+1}^{0} / \kappa_{N}^{0}$ (circles), $\kappa_{N+1}^{1} / \kappa_{N}^{0}$ (triangles), and $\kappa_{N+1}^{1} / \kappa_{N}^{1}$ (squares) as a function of $N$. 
be obtained by observing that $\kappa_{N+1}^{1} / \kappa_{N}^{0}$ has a constant behavior. For $\kappa_{4}^{1} / \kappa_{3}^{0}$ the universal ratio of 1.002 can be used, which was calculated in Ref. [11] in the zero-range theory, making a detailed numerical analysis of the solution of the Faddeev-Yakubovsky equations (for comparison, our numerical results obtained with the TBG potential give a value around 1.05). This analysis complete the determination of the ratios given in Eq. (12).

Finally, Eqs. (2) can be used to determine the relation between $\kappa_{N}^{m}$ and $a$ at different thresholds $\xi=-\pi$ at which the $N$-body cluster disappears in the $N$-body continuum. Using the notation $a_{N}^{m,-}$ for the corresponding value of $a$, in the zero-range limit we have

$$
\kappa_{N}^{m} a_{N}^{m,-}=-e^{-\Delta(-\pi) / 2 s_{0}} \approx-1.56
$$

This equation is an extension of the already known relation obtained in the zero-range $N=3$ case. In the case of finiterange interactions we use the working equations (6) and verify that the following modification of Eq. (16) is valid:

$$
\kappa_{N}^{m} a_{N}^{m,-} \approx-1.56-\Gamma_{m} .
$$

Here $a_{N}^{m,-}=a_{B}(-\pi)-a_{B}^{m}$, with $a_{B}(-\pi)$ being the value at the threshold determined either from direct calculations or from a global linear fit. We evaluate $\kappa_{N}^{m} a_{N}^{m,-}$ using the calculated values for $\kappa_{N}^{m}$ and $a_{N}^{m,-}$. The results are given in Fig. 7 for both $m=0$ and 1 . One can see that in both cases the quantity $\kappa_{N}^{m} a_{N}^{m,-}$ is close to the expected values of $-2.33(m=0)$ and $-2.45(m=1)$. For $m=0, \kappa_{N}^{m} a_{N}^{m,-}$ is better represented by a constant supporting the global fit. For $m=1$ the quantity $\kappa_{N}^{m} a_{N}^{m,-}$ deviates from a constant within $10 \%$ (given by the shadowed area) since the excited states' binding energies have not completely converged. In particular,

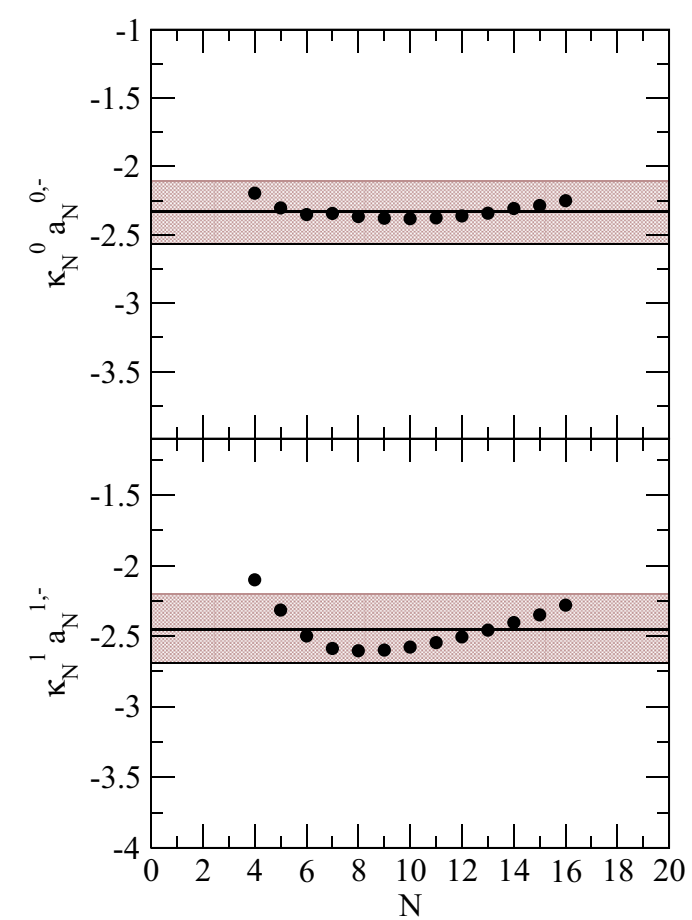

FIG. 7. (Color online) Dimensionless quantity $\kappa_{N}^{n} a_{N}^{n,-}$ as a function of $N$ for $n=0$ (top) and $n=1$ (bottom). the global fit for the excited states suffers from the fact that close to the $y(-\pi)$ threshold they are no longer bound for $N>7$. We have to mention here that in Ref. [16] a different parametrization of $a_{N}^{0,-}$ exists that involves four parameters. Such a parametrization does not show universality.

In the case of positive values of $a$, thresholds appear when the $N$-body systems disappears into the continuum formed by the different clusterization of the $N$-boson system. The first threshold appears at the $(N-1)$-boson energy and it is formed by a cluster of $N-1$ bosons and one boson staying far apart. Other thresholds are formed by $N / 2$ dimers (for even values of $N$ ) or by $(N-1) / 2$ dimers plus a particle (for odd values of $N)$. In the case of $N=4$ a detailed study of the behavior across the dimer-dimer and trimer-atom thresholds has been done in Ref. [25]. With an increasing number of bosons the structure of the thresholds becomes more and more complicated. As discussed in the $N=3$ case, the validity of Eqs. (2) for each $N$ system is limited by values of $\xi$ between $\xi=-\pi$ and the appearance of the first threshold.

\section{ANALYSIS OF THE RESULTS IN DIFFERENT SYSTEMS}

Most of the studies of $N$-boson systems with large twobody scattering lengths have been done either experimentally (real systems) or by solving the Schrödinger equation using potential models for the two-boson interaction. For example, here we have used a TBG potential, however, many other calculations with different model or realistic potentials can be found in the literature. In all these cases the deviations from the prediction of zero-range theory due to the finiterange nature of two-body interactions cannot be ignored. The working equations (4) and (6) proposed here can be used to analyze results obtained elsewhere. In particular, the Eqs. (4b) and (6b) can be used to study the linear dependence between $y$ and $a_{B}$ [see Eq. (9)].

As the first example we analyze the binding energies of the system of three ${ }^{7} \mathrm{Li}$ bosons measured in Ref. [34] for different values of the ${ }^{7} \mathrm{Li}-{ }^{7} \mathrm{Li}$ scattering length. From the values of the two- and three-body binding energies given in that reference we compute $a_{B}$, the angle $\xi$, and the universal function $y(\xi)$. According to Eq. (9), the values of $y$ should depend linearly on $a_{B}$. The results plotted in Fig. 8 do show the expected linear behavior, thus providing further confirmation of the finiterange theory given by Eqs. (6). From the analysis of the straight line we extract the values $\kappa_{3}^{1}=1.61 \times 10^{-4} a_{0}^{-1}$ and $\Gamma_{3}^{1}=$ $4.95 \times 10^{-2}$ (see also Ref. [21]).

As the second example we analyze the results for boson clusters obtained at the unitary limit by von Stecher in Ref. [16] using Gaussian potential models and including three-body forces. Empirically, the results for $N=6,7,8$ in the $a<0$ region were parametrized in Ref. [16] as $E_{N}^{0} \approx$ $\left(\hbar^{2} / m\right)\left(\kappa_{N}^{0}\right)^{2}\left(x+c_{N} x^{b_{N}}\right) /\left(1+c_{N}\right)$, where $x=\left(a-a_{N}^{-}\right) / a$. In addition, the empirical relation $1 /\left(\kappa_{3}^{0} a_{N}^{-}\right) \approx 2.3(1)-N$ has been deduced from the numerical result and the ratios $\left(\kappa_{N}^{0} / \kappa_{3}^{0}\right)^{2}$ have been calculated up to $N=13$. The results from Ref. [16] cannot be plotted in the $\left(a_{B}, y\right)$ plane because the explicit values of $E_{2}$ and $\kappa_{3}^{0}$ were not given. Instead we can plot them in the $\left(\kappa_{3}^{0} a, y\right)$ plane. The essential characteristics of this plot, given in the top panel of Fig. 9, remain the same 


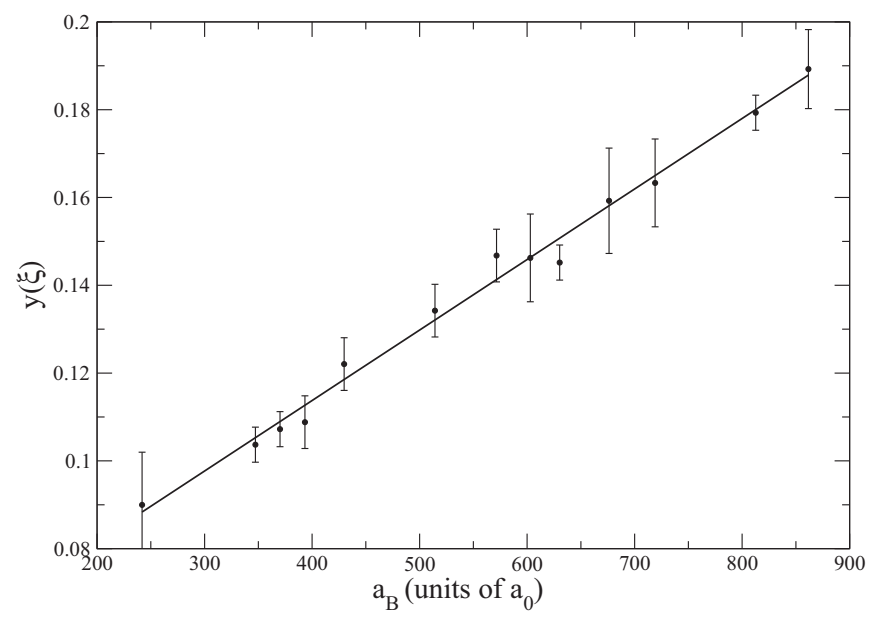

FIG. 8. Universal function $y(\xi)$ as a function of $a_{B}$ calculated from the data of Ref. [34]. The solid line represents the best linear fit to the data.

with the slope given by the ratio $\kappa_{N}^{0} / \kappa_{3}^{0}$ and the shift $\Gamma_{0}$ related by the linear equation (10). The results lie on straight lines, as predicted by the finite-range theory proposed here. Moreover, the straight lines seem to cross each other at one single point and accordingly can be described using a global fit with $\Gamma_{0} \approx-0.22$. This shift is negative and, in absolute value, it is slightly smaller than the value obtained here for $\Gamma_{0}$ in the global fit. In the bottom panel of the figure the ratio $\kappa_{N}^{0} / \kappa_{3}^{0}$ is
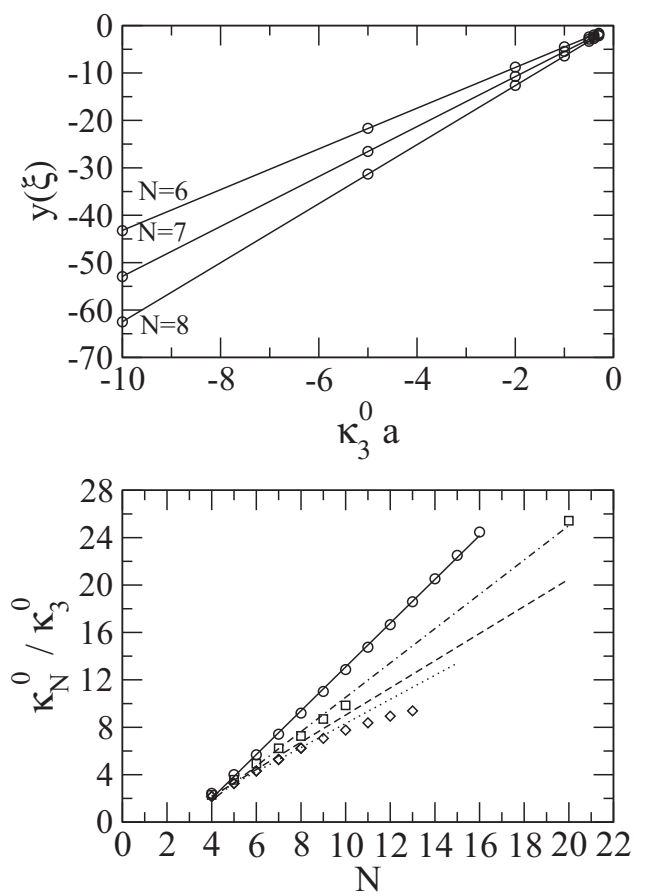

FIG. 9. Analysis of the results from Ref. [16] in the $(a, y)$ plane (top). In the bottom panel the ratios $\kappa_{N}^{0} / \kappa_{3}^{0}$ are given as a function of $N$ for the results of Ref. [16] (diamonds), the present results (circles), and the results from Ref. [35] (squares). The universal prediction is given by the dashed line. The solid and dash-dotted lines are fits to the data. The dotted line is a fit to the results of Ref. [16] up to $N=8$. given as a function of $N$. The diamonds are the results from Ref. [16]. At $N \leqslant 8$ they follow a linear behavior presented by a dotted line fitted over this region. They lie below the universal line $1+1.147(N-3)$ shown by the dashed line. On the other hand, our results, fitted by a solid line, are above the line of universality. The difference between our calculations and those from Ref. [16] is due to the absence of the repulsive three-body force in our case. With a two-body finite-range (Gaussian) force only, the $N$-boson clusters are more bound than in the zero-range case. Including three-body repulsive force reduces the strength of the linear dependence between $\kappa_{N}^{0} / \kappa_{3}^{0}$ and $N$ and eventually can reproduce the universal slope of 1.147. The particular three-body force selected in Ref. [16] produces a ratio slightly lower $\kappa_{4}^{0} / \kappa_{3}^{0}$ than the universal one and therefore the slope of the straight dotted line in the bottom panel of Fig. 9 is smaller, which corresponds to less bound clusters. The calculations of Ref. [16] deviate from the linear behavior (dotted lines) for $N>8$ and practically follow the square root law. This is discussed below.

The bottom panel of Fig. 9 also presents the results for $N$-boson clusters of polarized tritium obtained in Ref. [35] from a theoretical study within the diffusion Monte Carlo (DMC) method. In that work, the strength of the interaction was varied to explore a wide range of the $\left(a^{-1}, K\right)$ plane. The analysis of their results in the $\left(a_{B}, y\right)$ plane revealed that they lie on straight lines, as predicted by the finite-range theory. Here we show the linear dependence of the energy wave numbers on $N$ at the unitary limit. This is illustrated by the squares, whereas the dash-dotted line represents a linear fit to the data.

Finally we analyze two calculations from literature performed for the ground-state helium clusters with different numbers of $N$ using hard-core He-He potentials. From the published values of $E_{N}^{0}$, it is possible to determine the angle $\xi$ and from it the values of the universal function $y(\xi)$. From the previous discussion we expect a linear relation between $N$ and $y$, which means a quadratic dependence between $N$ and the ground-state energy. To perform this analysis we use the results from Lewerenz [36] and Pandharipande et al. [37]. In the former, the ground states up to ten atoms have been obtained within the DMC method with the Tang-Toennies-Yiu (TTY) potential [38] as the He-He interaction. In the latter, the Green's-function Monte Carlo (GFMC) method has been used with the HFDHE2 interaction of Aziz et al. [39]. The $y(\xi)$ extracted from these results are shown in Fig. 10, where the black circles and asterisks correspond to the DMC calculations with the TTY potential and the GFMC calculations with the HFDHE2 potential, respectively. We compare the hard-core results with those obtained with the soft-core TBG potentials in the present work, shown by squares. In addition, we show by triangles the $N \leqslant 6$ results from Ref. [28] obtained with the TBG potential plus a hyperradial three-body force (H3B) the strength of which is fitted to reproduce the trimer energy given by the LM2M2 potential. It should be noted that the TTY and the LM2M2 interactions produce very close results [40] for the helium dimer and trimer and therefore the results of the TBG+H3B interaction are almost on top of the results of the TTY potential. This supports the equivalence between the soft- and hard-core potentials for this kind of state, discussed, for example, in Ref. [41]. 


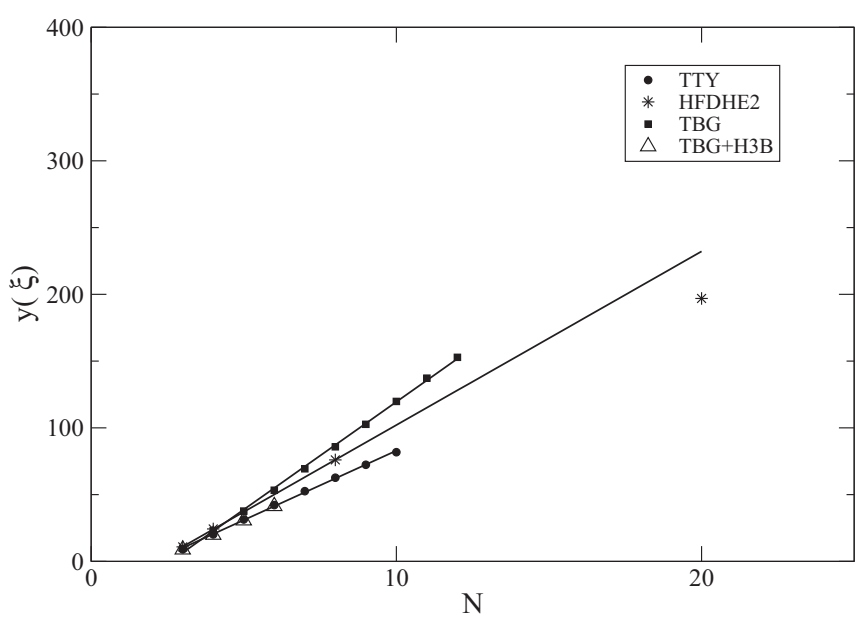

FIG. 10. Universal function $y(\xi)$ as a function of $N$ calculated using the results of Ref. [36] for the TTY potential (circles), the results of Ref. [37] for the HFDHE2 potential (asterisks), the present results for the TBG interaction (squares), and the results of Ref. [17] using a two-body plus a three-body force (triangles). The straight lines are the best fit to the data.

The above analysis shows that the finite-range-corrected universality relations proposed in the present work can be used to analyze different types of measurements and theoretical descriptions of shallow states in bosonic systems. However, we notice that the results obtained with the HFDHE2 potential and plotted in Fig. 10 no longer follow the linear behavior around $N=20$. They tend to follow a square root behavior since at $N \rightarrow \infty$ the ratio $E_{N}^{0} / N$ is almost constant, indicating the well-known linear dependence of the energy ground state with $N$. It is interesting to note that this analysis shows the transition between the quadratic and linear behavior of the ground-state energy with $N$. In the quadratic regime the bosons are far apart, the details of the interaction are not important, and the system shows the universal behavior described by Eqs. (6). As the number of bosons increases the system becomes more compact, losing the universal behavior. The strong short-range repulsion prevents the collapse of the system and is responsible for the linear regime. This explanation clarifies the behavior of the results from Ref. [16] for $N>8$. In this case a very-longrange repulsive three-body force has been included, making the transition to the linear regime already at low values of $N$. The observed transition from the quadratic to $E(N)$ linear behavior for the case of a realistic interaction with a (positive) large two-body scattering length is very interesting and should undergo deeper analysis in the future.

\section{CONCLUSION}

In the present work we have discussed DSI in shallow states of $N$-boson systems. We proposed an extension of the universal relation, known from three-body zero-range theory, to $N>3$ using the results of Refs. [9-11,17]. This was summarized in Eqs. (2), which show explicitly DSI and the two-level $N$-body structure attached to each level $n$ of the three-boson system. Then we extended Eqs. (2) to include corrections due to the finite-range nature of the two-body interactions. These corrections were encoded in the shift $\Gamma_{N}^{m}$, which we introduced into Eqs. (6) following Ref. [21]. At the origin of the extension of the universal equations to general $N$ is our finding that the universal function on the right-hand sides of Eqs. (6) that governs the three-boson dynamics is the same as the one that governs the $N$-boson dynamics. Such a conclusion was made by analyzing the solutions of the Schrödinger equations obtained in Refs. [17,21-23] with various model potentials.

The second striking property of the universal relations given in Eqs. (6) is the linear relation between $y(\xi)$, a particular form of the universal function, and $a_{B}$ (or $a$ in the zero-range theory). Using two-body short-range forces, the theory has two parameters: the slope and the distance along the $y$ axis to the origin given by the shift $\Gamma_{N}^{m}$. Following the analysis given for $N=3$, we proposed the zero-range theory for $N>3$ as the limit case $\Gamma_{N}^{n, m} \rightarrow 0$. The corresponding relations were given in Eqs. (2) and it could be seen that the zero-range limit can be represented by straight lines going through the origin and, accordingly, the theory has only one parameter: the slope.

We have checked if results available in the literature exhibit universal behavior given by Eqs. (6). We have plotted in the $\left(a_{B}, y\right)$ plane the experimental data on the energies of the ${ }^{7} \mathrm{Li}$ trimers, measured in Ref. [34], and proved that they show linear behavior. From the linear plot we extracted the values of $\kappa_{3}^{0}$ and $\Gamma_{0}$. At the unitary limit, we obtained $\kappa_{3}^{1}=1.61 \times 10^{-4} a_{0}^{-1}$, which can be checked either experimentally or theoretically. Furthermore, we have analyzed $N$-body energies calculated in Refs. [16,35], where a particular parametrization was proposed. However, we have shown that in both cases the linear behavior in the $\left(a_{B}, y\right)$ plane persists, thus restricting the number of parameters, needed to describe these systems, to two for each $N$. Moreover, those results show the expected linear dependence of the energy wave number on $N$ at the unitary limit. All these findings support both the zero-range and finite-range universal relations proposed here.

Further analysis showed that universal relations persist only for attractive two-body potentials. In real systems, a strong repulsion between atoms at short distances exists, which for fixed and (positive) finite values of $a_{B}$ leads to a transition to the square root behavior of the energy wave number with $N$. For the particular case of Ref. [37] this occurs around $N=20$. At larger $N$ the wave numbers cannot be described by the zero-range theory, which predicts a quadratic behavior of the ground-state energy with $N$. In this respect it would be interesting to study the boson clusters that have negative values of the two-body scattering length. In this case the shallow states are better realized and the transition between the quadratic and linear regime should occur at larger values on $N$. In fact, the results of Ref. [35] at the unitary limit show the transition to the quadratic regime at larger values of $N$ than those obtained in Ref. [37] for positive values of $a_{B}$. From a different perspective we would like to analyze the possibility of mimicking the different scale, arising due to the short-range repulsion, by introducing a four-body force. In this case a relation between the number of scales and high-order forces could be established. We hope that these findings will stimulate experimental and theoretical studies of $N$-body shallow states. 
[1] E. Braaten and H. Hammer, Phys. Rep. 428, 259 (2006).

[2] V. Efimov, Phys. Lett. B 33, 563 (1970).

[3] V. Efimov, Yad. Fiz. 12, 1080 (1970) [Sov. J. Nucl. Phys. 12, 589 (1971)].

[4] F. Ferlaino and R. Grimm, Physics 3, 9 (2010).

[5] M. Zaccanti et al., Nat. Phys. 5, 586 (2009); S. Roy, M. Landini, A. Trenkwalder, G. Semeghini, G. Spagnolli, A. Simoni, M. Fattori, M. Inguscio, and G. Modugno, Phys. Rev. Lett. 111, 053202 (2013).

[6] F. Ferlaino et al., Few-Body Syst. 51, 113 (2011).

[7] M. Berninger, A. Zenesini, B. Huang, W. Harm, H.-C. Nägerl, F. Ferlaino, R. Grimm, P. S. Julienne, and J. M. Hutson, Phys. Rev. Lett. 107, 120401 (2011).

[8] L. Platter, H.-W. Hammer, and U.-G. Meißner, Phys. Rev. A 70, 052101 (2004).

[9] H.-W. Hammer and L. Platter, Eur. Phys. J. A 32, 113 (2007).

[10] J. von Stecher, J. P. D'Incao, and C. H. Greene, Nat. Phys. 5, 417 (2009).

[11] A. Deltuva, R. Lazauskas, and L. Platter, Few-Body Syst. 51, 235 (2011).

[12] T. Frederico, L. Tomio, A. Delfino, M. R. Hadizadeh, and M. T. Yamashita, Few-Body Syst. 51, 87 (2011).

[13] H.-W. Hammer and L. Platter, Annu. Rev. Nucl. Part. Sci. 60, 207 (2010).

[14] L. Platter, Few-Body Syst. 43, 155 (2008).

[15] M. R. Hadizadeh, M. T. Yamashita, L. Tomio, A. Delfino, and T. Frederico, Phys. Rev. Lett. 107, 135304 (2011).

[16] J. von Stecher, J. Phys. B 43, 101002 (2010).

[17] M. Gattobigio, A. Kievsky, and M. Viviani, Phys. Rev. A 86, 042513 (2012).

[18] A. C. Phillips, Nucl. Phys. A 107, 209 (1968).

[19] J. A. Tjon, Phys. Lett. B 56, 217 (1975).
[20] M. Gattobigio, A. Kievsky, and M. Viviani, Few-Body Syst. 54, 1547 (2013).

[21] M. Gattobigio and A. Kievsky, Phys. Rev. A 90, 012502 (2014).

[22] A. Kievsky and M. Gattobigio, Phys. Rev. A 87, 052719 (2013).

[23] E. Garrido, M. Gattobigio, and A. Kievsky, Phys. Rev. A 88, 032701 (2013).

[24] H. E. Stanley, Rev. Mod. Phys. 71, S358 (1999).

[25] A. Deltuva, Few-Body Syst. 54, 569 (2013).

[26] R. A. Aziz and M. J. Slaman, J. Chem. Phys. 94, 8047 (1991).

[27] N. K. Timofeyuk, Phys. Rev. C 78, 054314 (2008).

[28] M. Gattobigio, A. Kievsky, and M. Viviani, Phys. Rev. A 84, 052503 (2011).

[29] N. K. Timofeyuk, Phys. Rev. A 86, 032507 (2012).

[30] A. O. Gogolin, Ch. Mora, and R. Egger, Phys. Rev. Lett. 100, 140404 (2008).

[31] S. Endo, P. Naidon, and M. Ueda, Phys. Rev. A 86, 062703 (2012).

[32] A. Kievsky, M. Gattobigio, and N. K. Timofeyuk, Few-Body Syst. 55, 945 (2014).

[33] A. N. Nicholson, Phys. Rev. Lett. 109, 073003 (2012).

[34] N. Gross et al., C. R. Phys. 12, 4 (2011).

[35] G. J. Hanna and D. Blume, Phys. Rev. A 74, 063604 (2006).

[36] M. Lewerenz, J. Chem. Phys. 106, 4596 (1997).

[37] V. R. Pandharipande, J. G. Zabolitzky, S. C. Pieper, R. B. Wiringa, and U. Helmbrecht, Phys. Rev. Lett. 50, 1676 (1983).

[38] K. T. Tang, J. P. Toennies, and C. L. Yiu, Phys. Rev. Lett. 74, 1546 (1995).

[39] R. A. Aziz, V. P. S. Nain, J. S. Carley, W. L. Taylor, and G. T. McConville, J. Chem. Phys. 70, 4330 (1979).

[40] P. Barletta and A. Kievsky, Phys. Rev. A 64, 042514 (2001).

[41] A. Kievsky, E. Garrido, C. Romero-Redondo, and P. Barletta, Few-Body Syst. 51, 259 (2011). 